\title{
Empoderamiento de mujeres con niños/as menores de cinco años y seguridad alimentaria - Distrito de Huacrapuquio
}

\section{Empowerment of women with children under five years old and food security - Huacrapuquio District}

\author{
'Scarsi Maratuech, R.M.; Huamán Quispe, R.; \\ 2Habich Scarsi, B.; Pérez Gutarra, G. \\ Facultad de Enfermería / Universidad Nacional del Centro del Perú \\ Email: rscarsi@uncp.edu.pe
}

\section{Resumen}

Empoderamiento de la mujer y seguridad alimentaria, son temas de gran interés en diferentes escenarios mundiales en el marco de los Objetivos de Desarrollo Sostenible; existiendo aún, vacíos en cuanto al conocimiento de ambos temas que podrían coadyuvar a fortalecerlos. El estudio correlacional tiene como objetivo general, el determinar la relación que existe entre empoderamiento de mujeres con niños/as menores de cinco años y seguridad alimentaria, se desarrolla con una población total de 57 madres pertenecientes al Programa del Vaso de Leche - PVL del distrito de Huacrapuquio (Junín-Perú).

La recolección de datos, para ambas variables, se realizó a través de la encuesta cuyos cuestionarios fueron aplicados individualmente a la población de estudio; los resultados demuestran que existe una relación directa entre el empoderamiento de mujeres y seguridad alimentaria, la mayoría de madres puntúan niveles bajos en ambas variables. Los hallazgos sugieren que el empoderamiento de mujeres puede facilitar la implementación de procesos orientados a la seguridad alimentaria, proponiendo a los actores claves de los escenarios afines la implementación de políticas públicas y estrategias participativas para potenciar el empoderamiento de mujeres y la seguridad alimentaria; considerando, además, estudios futuros para establecer posibles relaciones causales.

Palabras Clave: empoderamiento de mujeres, seguridad alimentaria, género, salud.

\begin{abstract}
Empowerment of women and food security are topics of great interest in different global scenarios within the framework of the Sustainable Development Goals, there are still gaps in knowledge of both issues that could help strengthen them. The general objective of the correlational study is to determine the relationship between the empowerment of women with children under the age of five and food security, a total population of 57 mothers belonging to the Social Milk Glass Program (Programa de Vaso de Leche - PVL) of Huacrapuquio District (Junin-Peru) was developed.

The data collection for both variables has been done through the survey whose questionnaires were applied individually to the study population; results show that there is a direct relationship between the empowerment of women and food security, most mothers score low levels in both variables. Findings suggest that empowerment of women can facilitate the implementation of processes oriented to food security, suggesting the implementation of public policies and participatory strategies to enhance the empowerment of women and food security to key actors of related scenarios, also considering future studies to establish possible causal relationships.
\end{abstract}

Keywords: women empowerment, food security, gender, health. 


\section{Introducción}

A través de la historia, la mujer ha sido catalogada como una persona que cumple solamente roles domésticos, dejando de ser valorada en las diferentes sociedades; no obstante, es de conocimiento que su rol básico es de velar por el bienestar y seguridad de la familia y, dentro de ella, de los hijos, para que puedan crecer y desarrollar sus potencialidades adecuadamente y ser ciudadanos que puedan aportar al desarrollo del país.

Es así, que en el marco de la definición de los Objetivos de Desarrollo Sostenible y teniendo como referencia la Cuarta Conferencia Mundial sobre la Mujer, existe el consenso mundial para implementar políticas y estrategias orientadas a la igualdad de género, siendo el empoderamiento de las mujeres una de las prioridades en todos los espacios; sin embargo, no se cuenta con avances tangibles, siendo necesario una agenda conjunta e integral considerando los derechos humanos (ONU Mujeres, 2016).

En el escenario, el empoderamiento de mujeres, es considerado como la participación plena en espacios sociales, económicos, políticos, familiares e individuales que les permitan configurar sus propias vidas y de su entorno, para coadyuvar a su desarrollo individual y colectivo.

Lo mencionado implica que el empoderamiento de mujeres es lograr que puedan participar activamente en la toma de decisiones individuales y colectivas a través del desarrollo de sus competencias, lo cual además de otros aspectos puede permitir fortalecer el despliegue de sus capacidades y funciones, la seguridad alimentaria y por ende el adecuado desarrollo y crecimiento de sus hijos.

Pero, a pesar de la importancia que se otorga al empoderamiento de mujeres y seguridad alimentaria, existen pocas investigaciones que se han desarrollado sobre la relación de ambas variables.

Con estas consideraciones, el estudio tuvo como objetivo el determinar la relación entre empoderamiento de mujeres con niños/as menores de cinco años y seguridad alimentaria; así como, investigar la relación que existía entre dimensiones seleccionadas del empoderamiento y la seguridad alimentaria.

El estudio permitió generar nuevos conocimientos en cuanto a la relación entre empoderamiento de la mujer y la seguridad alimentaria; asimismo, incentivó el interés para otras investigaciones, proponiéndo a los directivos, de los diferentes escenarios afines, la generación de políticas públicas y estrategias participativas orientadas al fortalecimiento del empoderamiento de mujeres y seguridad alimentaria.

\section{Materiales y métodos}

\section{Población}

Este estudio correlacional, se realizó a través de la encuesta a una población de 57 madres con niños/as menores de cinco años que pertenecían al Programa del Vaso de Leche del distrito de Huacrapuquio (Huancayo - Perú) en el segundo semestre del 2017.

\section{Instrumentos}

Para la recolección de datos, se utilizó como técnica la encuesta a través del cuestionario denominado Empoderamiento de la Mujer (IMEN), elaborado por Hernández y García (2008), el cual contiene siete dimensiones: Empoderamiento participativo, temeridad, influencias externas, igualdad, satisfacción social y seguridad; siendo medidos a través de 34 ítems que mostraron buena fiabilidad.

Para la variable seguridad alimentaria, se utilizó la Escala de la Inseguridad Alimentaria - Hogar (acrónimo en inglés: FIES-SM), formulada por la Organización de las Naciones Unidas para la Agricultura y Alimentación (acrónimo en inglés - FAO), el cuestionario contiene 8 ítems, los cuales se analizaron en conjunto, mostrando una buena fiabilidad. (FAO, 2014).

\section{Diseño y procedimiento}

Al ser un estudio de tipo correlacional trasversal, respondió al siguiente esquema:

Dónde:

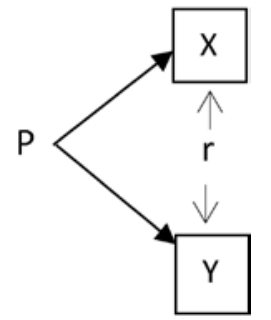

$\mathrm{P}=$ Población.

$\mathrm{X}=$ Conjunto de datos con respecto a empoderamiento

$\mathrm{Y}=$ Conjunto de datos con respecto a seguridad alimentaria

$r=$ Relación existente entre ambas variables.

La recolección de datos, para ambas variables, se realizó a través de la encuesta a una población de $57 \mathrm{mu}$ jeres que tenían niños/as menores de cinco años y que pertenecían al Programa del Vaso de Leche del distrito de Huacrapuquio en el segundo semestre del año 2017.

La responsable del Programa del Vaso de Leche, fue contactada previamente por los investigadores obteniéndose la autorización para realizar el estudio. A las madres se les explicó los objetivos de la investigación y los alcances respectivos, procediéndose a 
aplicar los cuestionarios impresos en papel, los que fueron respondidos en forma individual en el ambiente del Programa del Vaso de Leche durante el horario de su reunión.

La aplicación de los cuestionarios fue realizada y supervisada por los investigadores, como criterio de exclusión se consideró a las madres que no estaban presentes al momento de aplicarlos y aquellas que no deseaban participar en el estudio, 10 madres no asistieron quedando la población conformada por 57 madres. El proceso tuvo en cuenta las consideraciones éticas nacionales e internacionales.

\section{Análisis de datos}

Para realizar el análisis de datos, se trabajó con el programa estadístico SPSS versión 24. Se aplicó la estadística descriptiva, las tablas simples se usaron para medir el empoderamiento de mujeres, sus dimensiones y la medición de la seguridad alimentaria. Se usó las tablas de doble entrada para medir la relación entre el empoderamiento y la seguridad alimentaria.

Se hizo uso de la estadística inferencial, debido a que las variables eran ordinales, aplicándose la prueba Tau b de Kendall para medir la relación que existe entre el empoderamiento y la seguridad alimentaria; así mismo, la relación de las dimensiones del empoderamiento y la seguridad alimentaria.

\section{Resultados}

Los resultados encontrados en el estudio no fueron muy alentadores por los bajos niveles de empoderamiento y seguridad alimentaria.

Los hallazgos del estudio, de acuerdo a la Tabla 1, mostraron que el $73.7 \%$ de mujeres percibian que tenian un nivel de empoderamiento bajo, el $26.3 \%$ un nivel medio, no encontrandose nivel alto.

Tabla 1

Nivel de empoderamiento de mujeres

\begin{tabular}{lccc}
\hline Empoderamiento & Frecuencia & Porcentaje & $\begin{array}{c}\text { Porcentaje } \\
\text { acumulado }\end{array}$ \\
\hline Bajo & 42 & 73,7 & 73,7 \\
Medio & 15 & 26,3 & 100,0 \\
Total & 57 & 100,0 & \\
\hline
\end{tabular}

Nota. Nivel expresado en (\%).

Los resultados del estudio, en relación a la seguridad alimentaria, de acuerdo a la Tabla 2, evidenciaron que el $71.9 \%$ consideraban que era baja, el $22.8 \%$ media y solo el $5.3 \%$ alta.
Tabla 2

Nivel de seguridad alimentaria

\begin{tabular}{lccc}
\hline $\begin{array}{c}\text { Seguridad } \\
\text { alimentaria }\end{array}$ & Frecuencia & Porcentaje & $\begin{array}{c}\text { Porcentaje } \\
\text { acumulado }\end{array}$ \\
\hline Baja & 41 & 71,9 & 71,9 \\
Media & 13 & 22,8 & 94,7 \\
Alta & 3 & 5,3 & 100,0 \\
Total & 57 & 100,0 & \\
\hline
\end{tabular}

Nota. Nivel expresado en porcentaje (\%).

Los hallazgos encontrados en la Tabla 3, demostraron que existe una relación directa, estadísticamente significativa, entre el empoderamiento de mujeres con niños/as menores de cinco años y seguridad alimentaria. $(0,627)$, significativa $(p=0,000>0,05)$.

Tabla 3

Correlación entre empoderamiento y seguridad alimentaria

\begin{tabular}{cccc}
\hline & Tau b de Kendall & $\begin{array}{c}\text { Empodera } \\
\text {-miento }\end{array}$ & $\begin{array}{c}\text { Seguridad } \\
\text { alimentaria }\end{array}$ \\
\hline $\begin{array}{c}\text { Empodera- } \\
\text { miento }\end{array}$ & Sig. (unilateral) &. &, $627 * *$ \\
& $\mathrm{~N}$ & 57 &, 000 \\
& Tau b de Kendall &, $627 * *$ & 57 \\
$\begin{array}{c}\text { Seguridad } \\
\text { alimentaria }\end{array}$ & Sig. (unilateral) &, 000 & 1,000 \\
& $\mathrm{~N}$ & 57 & 57 \\
\hline Nota. ** La correlación es significativa en el nivel 0,01 (bilateral)
\end{tabular}

En cuanto a la relación entre cada una de las dimensiones del empoderamiento de mujeres y seguridad alimentaria, los resultados mostraron:

Entre empoderamiento dimensión participativa y seguridad alimentaria, no existía una relación estadísticamente significativa entre ambas variables. $(-0,151)$, significativa $(\mathrm{p}=0,233<0,05)$.

Entre empoderamiento dimensión temeridad y seguridad alimentaria, no existía una relación directa $(0,018)$, significativa $(\mathrm{p}=0,891<0,05)$.

Entre empoderamiento dimensión influencias externas y seguridad alimentaria, no existía una relación directa $(0,023)$, significativa $(p=0,855<0,05)$.

Entre empoderamiento dimensión independencia $\mathrm{y}$ seguridad alimentaria, no existía una relación directa $(0,126)$, significativa $(\mathrm{p}=0,321<0,05)$.

Entre empoderamiento dimensión igualdad y seguridad alimentaria, existía una relación directa estadísticamente significativa $(0,289)$, significativa $(p=0,026<0,05)$.

Entre empoderamiento dimensión satisfacción social y seguridad alimentaria, existía una relación directa $(0,177)$, significativa $(p=0,169<0,05)$. 
Entre empoderamiento dimensión seguridad y seguridad alimentaria, existía una relación directa estadísticamente significativa $(0,296)$, significativa $(p=0,020<0,05)$.

\section{Discusión}

De acuerdo con Aranda et al.(xxxx), el empoderamiento de mujeres es considerado como la participación plena en espacios sociales, económicos, políticos, familiares e individuales que les permita configurar sus propias vidas y de su entorno, para coadyuvar a su desarrollo individual y colectivo. Sin embargo, en las mujeres estudiadas, se encontró que el 26.3 \% tenían una percepción media del empoderamiento y el $73.7 \%$ baja, resultados similares a los encontrados por Chávez (2011), quien determinó que en todas las dimensiones del empoderamiento, las mujeres presentaban mayor proporción en los niveles bajos y medios, a pesar que en el marco de políticas mundiales la mujer tiene un rol importante para la toma de decisiones, lo cual no es tomado en cuenta en muchos escenarios, corroborando lo que menciona Climént (2009), que la controversia que existe es que, a través de la historia la mujer ha sido relegada y no valorada en las diferentes sociedades, no considerándose el rol importante que cumple en el cuidado de los hijos, y su aporte al desarrollo de los diferentes espacios donde se desenvuelve, aunado a que se encuentran indicadores a través del tiempo, relacionados con desigualdad de género como: autonomía de las mujeres, acceso a la educación y acceso al trabajo.

Los resultados de la investigación, en cuanto a la percepción de la dimensión empoderamiento participativo, evidenciaron que el $17.5 \%$ presentaban un nivel alto, el $70.2 \%$ medio y el $12.3 \%$ bajo. La calificación baja demostró características en que las mujeres tenían pocas capacidades para liderar, escasas oportunidades de participación en actividades emprendedoras y autosuficientes, considerándose una actitud pasiva, poco activista y tal vez tímida. Concordando con Zapata et al. (2002), quien menciona que es necesario que las mujeres faciliten su empoderamiento como un proceso individual que logre generar escenarios para su fortalecimiento.

En la dimensión temeridad, el $94.7 \%$ presentaban un nivel bajo y $5.3 \%$ medio, lo que nos hizo inferir que las mujeres para participar políticamente tienen que negociar con su pareja considerando que es mejor que sea el hombre el que dirige, sintiéndose inseguras al tomar decisiones y el pensar que para ser líder se tiene que nacer con cualidades. Al respecto, Casique (2010), demostró que el empoderamiento de mujeres se ha considerado como un aspecto que abarca procesos y resultados que permitan mayor accesibilidad para el logro de competencias y acceso a recursos.

En cuanto a la dimensión influencias externas, el 17.5\% presentaban percepción baja, $73.7 \%$ medio y el $8.8 \%$ alto. Las mujeres con niveles bajos, consideraban que el liderazgo depende de otras personas y que es una habilidad que no la tenían y que no podían ocupar cargos de poder o políticos.

La dimensión independencia, reflejaba que el $63.2 \%$ de mujeres tenían una percepción baja, lo que significaba que ellas consideraban que siempre sus parejas debían saber dónde se encontraban, se esmeraban por cumplir las expectativas de sus seres queridos a costa de lo que ellas realmente querían y que su felicidad dependía de la felicidad de los demás. Por lo que se puede asumir que, este grupo de mujeres mostraban una gran dependencia a su pareja, familia y otros agentes externos, en contraposición a las mujeres que tiene niveles altos $8.7 \%$ y asumen su capacidad para decidir. Al respecto Kabeer (1999), encontró en su estudio, que el empoderamiento está ligado a la toma de decisiones, otorgándose poder para participar activamente en lo que anteriormente no estaba al alcance.

Así mismo, el $1.8 \%$ de mujeres mostraban una percepción alta, el $14 \%$ media y el $84.2 \%$ baja en relación al empoderamiento en su dimensión de igualdad, resultados alarmantes que indicaban que la mayoría de ellas percibían que no tenían las mismas oportunidades que los hombres para acceder a puestos de toma de decisiones y que ellos tenían más derechos.

En la dimensión satisfacción social el 75.4\% de mujeres tenían una percepción baja, $21.1 \%$ media, lo que significaba que las mujeres percibían poco reconocimiento de su entorno familiar y colectivo disminuyendo su confianza en sí mismas. En contraposición a las mujeres que tenían percepción alta (3.5\%), mencionado los resultados de Rodríguez (2015), en que las mujeres aceptaron que a partir de sus necesidades tienen las competencias para rediseñarse como agentes sociales y fortalecer su autonomía.

El $75.5 \%$ de mujeres mostraban una percepción baja y el $14 \%$ media en la dimensión seguridad, lo que denota que percibían poco reconocimiento de su entorno familiar y colectivo, disminuyendo su confianza en sí mismas, estos resultados pueden estar relacionados a que la baja seguridad en las mujeres está asociada a los roles domésticos que tienen las mujeres a través de la historia, como a otros factores (Matud, 2004), en contraposición al $10.5 \%$ de mujeres que mostraban niveles altos y percibían seguridad en sí mismas, en sus competencias, en su rol, en la relevancia de sus acciones y decisiones; para así mejorar su situación en relación a derechos sociales políticos, actividad econó- 
mica y productiva, para mejorar su calidad de vida y de su familia. Estos resultados permitieron analizar en base a resultados de un estudio que existen reflexiones más amplias sobre la importancia de la transferencia de políticas para organizar y contabilizar las actividades de gobernabilidad y empoderamiento (Demeritt, Rothstein, Beaussier, \& Howard, 2015).

Así mismo, considerando el análisis del empoderamiento de mujeres líderes políticas a través de sus hechos de vida, se demostró que a pesar de ser de diferentes orígenes comparten prácticas que facilitan u obstaculizan su capacidad de empoderamiento. (Hernández \& García, 2015).

En relación a la seguridad alimentaria, tomando como referencia la definición de la FAO (2009), es considerada como la accesibilidad a alimentos sanos y nutritivos, que satisfagan los requerimientos para una vida saludable, sobre todo de los niños/as; sin embargo, los resultados del estudio mostraron que solo el 5,3\% de mujeres percibían un nivel de seguridad alimentaria alto, el $22.8 \%$ medio y el $71.9 \%$ bajo, habiéndose demostrado que existe relación con el empoderamiento, resultados similares a los encontrados por Shuai et al. (2018), quien determinó que el empoderamiento tenía un efecto significativo en los mecanismos de vida en el hogar, en la misma línea los resultados indican que el empoderamiento de las mujeres influye positivamente en la disponibilidad de nutrientes (Tsiboe, 2018).

\section{Conclusiones}

- Tal y como evidenciaron los resultados de la investigación, en el que se demostró bajos niveles de percepción de las mujeres sobre su empoderamiento y seguridad alimentaria, se infiere que puede deberse al paradigma social, en que la mujer a través de los años fue excluida y tuvo que soportar la percepción de inferioridad e injusticia social, coadyuvado a que muchas veces las decisiones que afectan a su persona y su familia son tomadas por otros, sin considerar su filosofía, su lenguaje, simbología, sueños y aspiraciones, quitándoseles el derecho de ser partícipes de su propio desarrollo influyendo en su calidad de vida y de su familia.

- En ese contexto, es evidente el escaso empoderamiento de las mujeres, a pesar de las diferentes políticas emanadas desde los diferentes escenarios que tienen como principal derrotero la participación COmunitaria y social, siendo un agente importante de ello la mujer.

- Así mismo, los resultados demostraron la difícil situación de las mujeres para acceder a alimentos que permitan asegurar la adecuada nutrición de sus hijos. Las causas principales están referidas a la dis- ponibilidad de alimentos, inaccesibilidad económica, cultural y física de los mismos; jugando un rol preponderante el nivel de educación de la madre. Al respecto, se puede mencionar que el $35.1 \%$ de las mujeres encuestadas solo tenían educación primaria, lo cual limita sus competencias, al respecto Baig et al. (2017), en su investigación comprobó, estadísticamente, que la toma de decisiones y la autoestima son factores significativos que permiten logros favorables en el desarrollo rural y que una buena educación y posibilidades laborales podían influir en el empoderamiento de mujeres.

- Además, el empoderamiento y seguridad alimentaria supone que las mujeres actúen individualmente y colectivamente con el fin de conseguir la seguridad alimentaria de su familia y sobre todo de sus hijos, siendo este un objetivo principal para generar políticas públicas y estrategias participativas a nivel local, regional y nacional, que respondan a las principales necesidades del entorno.

- Por otro lado, los resultados demostraron que entre empoderamiento y sus dimensiones: participativa, temeridad, influencias externas, independencia y satisfacción social no existía una relación con la seguridad alimentaria. En cuanto a las dimensiones igualdad y seguridad si existía una relación con la seguridad alimentaria.

\section{Referencias bibliográficas}

Baig, IA, Batool, Z., Ali, A., Baig, SA, Hashim, M. y Zia-ur-Rehman, M. (2017). Impacto del empoderamiento de las mujeres en el desarrollo rural en el sur de Punjab, Pakistán. Calidad y Cantidad, 52 (4), 1861-1872. doi: 10.1007 / s11135-017-0572-x

Bayo, N., \& Maya Jariego, I. (2014). Participación comunitaria, empoderamiento y salud percibida de mujeres en el entorno rural de Sevilla - PDF. Apuntes de Psicología, 32(1), 65-76. Retrieved from http:// docplayer.es / 20001091-Participacion-comunitaria-empoderamiento-y-salud-percibida-de-mujeres-en-el-entorno-rural-de-sevilla.html

Cano, T., \& Arroyave, O. (2014). Procesos de empoderamiento de mujeres: subjetivación y transformaciones en las relaciones de poder. RevistaVirtual Universidad Católica Del Norte, (42), 94-110. Retrieved from

Casique, I. (2010). Factores de empoderamiento y protección de las mujeres contra la violencia. México D.F. UNAM, Revista Mexicana de Sociología, 72(1), 37-71.

Cediel Becerra, N. M., Donoso Burbano, N., Hernández Manzanera, J., López Duarte, M. C., Herrera Buitrago, P., \& Moreno González, C. (2017). Em- 
poderamiento de las mujeres rurales como gestoras de los Objetivos de Desarrollo Sostenible en el posconflicto colombiano. Equidad y Desarrollo. https: / /doi.org/10.19052/ed.4077

Climent, G. (2009). Voces, silencios y gritos: Los significados del embarazo en la adolescencia y los estilos. Revista Argentina de Sociología, 7, 186-213.

Demeritt, D., Rothstein, H., Beaussier, A. L., \& Howard, M. (2015). Mobilizing risk: Explaining policy transfer in food and occupational safety regulation in the UK. Environment and Planning A, 47(2), 373-391. https://doi.org/10.1068/a140085p

Organización de las Naciones Unidas para la Alimentación y la Agricultura. (2009). El estado de la inseguridad alimentaria en el mundo. SOFI 2009. Roma

Organización de las Naciones Unidas para la Alimentación y la Agricultura. Proyecto Voces del Hambre - Escala de inseguridad alimentaria basada en la experiencia - Modulo de la encuesta [Internet]. Roma: FAO, 2014: 1-2 [citado nov 15, 2016]. Disponible en: http://www.fao.org/3/a-bl404s.pdf

Chávez L. (2011) Nivel de empoderamiento en el hogar de las madres de niños menores de cinco años que asisten al centro de salud "San Juan de Miraflores" (Tesis de Pregrado) Universidad Mayor de San Marcos, Lima.

Hernández, J. (2008). Instrumento para medir el empoderamiento de la mujer. Tabasco, Mexico.

Hernández Sánchez, J. E., \& García Falconi, R. (2015). Empoderamiento en mujeres mexicanas: Experiencias de mujeres líderes de México. Cuestiones de género: de la igualdad y la diferencia, 10, 419-434. https: / / doi.org/10.18002/cg.v0i10.1390

Instituto Nacional de Estadística e Informática. (2014). Resumen ejecutivo: Encuesta demográfica y de salud familiar, Lima.

Instituto Nacional de Estadística e Informática. (2015). Condiciones de vida en el Perú, Informe técnico N. ${ }^{\circ} 2$ (junio 2015, enero, febrero y marzo 2015).

James P, Tombs S, Whyte D, (2013), “An independent review of British health and safety regulation? From common sense to non-sense" Policy Studies

Kabeer, N. (1999). Resources, agency, achievements: reflections on the measurement of women's empowerment. Development and Change, 30, 435-464.
Kuttab, E. (2010). El empoderamiento como resistencia: conceptualizando el empoderamiento de las mujeres palestinas. Development, 53(2), 247-253. https: / / doi.org/10.1057/dev.2010.22

Lamer W, Laurie N, (2010). Travelling technocrats, embodied knowledges: globalising privatisationin telecoms and water. Geoforum

Lofstedt R, (2011). Reclaiming health and safety for all: an independent. Review of Health and Safety Legislation (Stationery Office, London) 